Originalveröffentlichung in: Sabatier, Gérard ; Torrione, Margarita (Hrsgg.): ¿ Louis XIV espagnol ? Madrid et Versailles, images et modèles. Paris 2009, S. 75-93

\title{
LE LiON ET LE GLOBE : LA STATUE DE LOUIS XIV par Domenico Guidi, ou l'EsPagne humiliée
}

\author{
Hendrik Ziegler*
}

Louis XIV n'a cessé de considérer l'Espagne comme l'ennemie de la France. Dans ses Mémoires, instructions pour gouverner conçues à l'intention du Dauphin, il ne manqua pas d'évoquer la relation de concurrence jalouse et d'" inimitié permanente " entre les deux peuples, qui n'aurait jamais su être entièrement éliminée en dépit de son occultation passagère par des traités politiques et des mariages dynastiques ${ }^{1}$. Le changement de rapport de force à partir des années 1650, confirmé par la paix des Pyrénées en 1659 qui allait durablement sceller la faiblesse militaire de l'Espagne, tarda à être reconnu du côté français : dans la perception des contemporains de Louis XIV, l'Espagne restait jusqu'au tournant du siècle un adversaire important et menaçant ${ }^{2}$. Pour cette raison, la politique étrangère ludovicienne continua de poursuivre parmi ses principaux objectifs l'humiliation diplomatique, la lutte militaire et l'amputation territoriale de l'Espagne, jusqu'à ce que le testament de Charles II attribue la couronne espagnole à la maison de Bourbon. Dans le domaine de la politique artistique, le conflit franco-espagnol demeura également ouvert tout au long de la seconde moitié du XVII siècle, ainsi qu’une statue de Louis XIV réalisée

* Je remercie Diane H. Bodart et Gérard Sabatier qui m’ont apporté des informations et des idées précieuses, et pour leur relecture attentive de la traduction.

1. Louis XIV, Mémoires pour l'instruction du Dauphin, édition P. Goubert, Paris, Imprimerie nationale, 1992, p. 70.

2. Jean-Frédéric Schaub, La France espagnole : les racines hispaniques de l'absolutisme français, Paris, Seuil, 2003, p. 210-211 et p. 316-317. 
à la fin des années 1690 à Rome le démontre de façon exemplaire. Par son message éminemment politique, elle anticipe en effet, grâce aux moyens de l'art, l'appropriation de la couronne d'Espagne par le roi de France.

En l'absence d'héritiers du faible Charles II, à la santé chancelante, le règlement de la succession d'Espagne était devenu de plus en plus urgent dans la seconde moitié des années 1690 , provoquant un nouvel affrontement entre

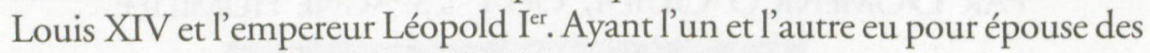
filles de la maison d'Espagne, les deux monarques étaient en droit de revendiquer le trône vacant pour l'un de leurs héritiers respectifs. Dans la phase préliminaire de ce conflit latent, Rome revêtit un rôle stratégique central : le pape Innocent XII, qui en raison de sa souveraineté spirituelle avait une fonction d'arbitre dans les conflits internationaux, était en outre, en sa qualité de suzerain du royaume de Naples, appartenant à la couronne d'Espagne, directement impliqué dans chaque règlement de succession. Par conséquent, la voie diplomatique fut d'abord tentée, à l'aide d'émissaires, non seulement à la cour de Madrid mais aussi auprès de la curie pontificale, afin de rallier des partisans aux solutions respectivement proposées par la France et l'Empire.

C'est dans ce climat tendu qu'en 1697 le prince Vaini, nouvel allié ambitieux du parti français à Rome, fit réaliser une statue en marbre de Louis XIV. Commencée par Domenico Guidi et sans doute achevée en 1699 par Pierre Legros le Jeune, elle affichait explicitement les ambitions impériales du Roi-Soleil et son intention d'établir sa domination sur l'Espagne, en le représentant foulant aux pieds le lion et le globe, attributs emblématiques de cette puissance. Une œuvre aussi provocante, de conception ouvertement antihabsbourgeoise et dirigée aussi bien contre l'Espagne que contre la maison mère d'Autriche, ne fut pas sans susciter de vives réactions diplomatiques. De récentes recherches documentaires permettent de confirmer que Georges Adam, comte de Martinitz, émissaire impérial à Rome entre 1695 et 1700 , alla jusqu'à proférer des menaces de mort à l'encontre de l'artiste.

\section{La commande de la statue par Guido Vaini}

On sait peu de chose sur Guido Vaini, décédé à Rome en $1720^{3}$. Au début de 1697, il bénéficia d'une importante promotion. Vaini possédait

3. Gabriel Hanotaux (dir.), Recueil des instructions données aux ambassadeurs et ministres de France depuis les traités de Westphalie jusqu'à la Révolution Française, t. XVII, $2^{\mathrm{e}}$ part. : Rome (1688-1723), Paris, Alcan, 1911, p. 143. 
plusieurs seigneuries dans les monts Sabins, qu'il avait acquises de la famille ducale des Cesi Di Acquasparta : le château de Cantalupo ainsi que les villages de Selci et de Gavignano. Quelque temps plus tard, le pape Innocent XII avait élevé Cantalupo au rang de principauté (Principato), de telle sorte que Vaini était désormais en droit de porter le titre de "Prince de Cantalupe et Duc de Selci, Marquis de Vacone, Seigneur de Gavignano ». Peu après, au mois de mars 1697, Vaini afficha publiquement son ralliement au parti francophile en faisant accrocher, selon une pratique courante, les armoiries de son nouveau protecteur Louis XIV audessus du portail de son palais romain ${ }^{4}$. Indigné, Martinitz rapporta immédiatement à l'empereur que le pape Innocent XII s'était réjoui du choix de Vaini, alors qu’à son avis sa joie n'eût probablement pas été aussi grande si le prince avait élu le parti impérial. La sympathie que le pape vouait à la faction française lui apparaissait ainsi évidente 5 .

L'engagement de Vaini pour la cause française s'avéra fort profitable. Le 2 février 1698, il fut fait par Louis XIV chevalier de l'ordre du Saint-Esprit, la plus haute distinction que le roi de France était en mesure d'accorder, et il prêta personnellement serment à Versailles un an plus tard, le 7 juin 1699. Parmi les démonstrations de loyauté de Vaini, qui devaient aboutir à son admission dans l'ordre et à une protection royale particulière, ne figure pas seulement l'apposition des armoiries fleurdelisées sur son palais mais également la réalisation d'une statue de marbre monumentale de Louis XIV, commandée en cette même année 1697 à Domenico Guidi et destinée au palais romain du prince ${ }^{6}$. En France, l'Italien Guidi n'était pas un inconnu : parmi les sculpteurs de son pays les plus appréciés par le roi, il s'était affirmé comme l'un des partenaires fiables de la France. En tant que vice-président de l'Accademia di San Luca, Guidi s'était engagé avec succès pour la réunion de l'Académie romaine avec l'Académie royale de peinture et de sculpture de Paris, officiellement ratifiée au début de l'année 1677.

4. Dietrich Erben, Paris und Rome: Die staatlich gelenkten Kunstbeziehungen unter Ludwig XIV, Berlin, Akademie-Verlag, 2004, p. 247, note 53.

5. Österreichisches Staatsarchiv, Vienne, section Haus-, Hof- und Staatsarchiv, Rome, correspondance, carton 78, dossier « 1697 », Georges Adam Martiniz à Léopold I er, Rome, 6 avril 1697 , fol. $103-106$.

6. Une note en date du 10 mai 1687 donne une première indication sur le travail réalisé Sur la statue; voir David L. Bershad, Domenico Guidi, a $17^{\text {th }}$ Century Roman Sculptor, PhD thesis, Los Angeles, University of California, 1971, p. 36-37, notes 43 et 47. 
couronné de lauriers et tenant dans sa main droite un bâton de commandement. Un pied sur un globe et l'autre sur une peau de lion, la connotation hispano-habsbourgeoise de ces attributs est évidente.

\section{Une iconographie volontairement anti-habsbourgeoise}

L'usage de conférer des attributs impériaux à Louis XIV, afin d'exprimer sa prétention au pouvoir universel, était à cette date désormais bien établi auprès des artistes de cour en France. L'une des stratégies les plus courantes était de s'approprier une iconographie souveraine traditionnellement associée à Charles Quint et à Philippe II, en la reconfigurant de façon créative. Ce procédé fut également mis en ouvre dans le cas de la statue romaine. Pourtant Vaini, par l'intermédiaire du sculpteur Guidi, était même allé plus loin en thématisant subtilement, grâce au langage figuratif, la faiblesse de la maison de Habsbourg, et en particulier de Charles II, et la relève espérée du règne de ce dernier par les Bourbons. Le globe placé sous le pied gauche de Louis XIV renvoyait clairement à l'attribut impérial qui, associé à la figure de Charles Quint, s'était imposé comme signe de domination universelle dans la représentation du souverain moderne. Le portrait triomphal de Charles Quint triomphateur et dominateur du monde, entouré d'une Victoire et d'un Hercule enfant lui apportant un globe, réalisé par Parmigianino en 1530 à l'occasion du couronnement de l'empereur à Bologne, avait inauguré la typologie du portrait allégorique de grand format de la Renaissance, en figurant l'empereur assis, prenant appui sur le globe ou le tenant entre ses mains ${ }^{10}$ (H.-T. 17).

De même - en réactualisant des formules figuratives antiques - le type de représentation de l'empereur posant un pied sur le globe avait resurgi au cours de la Renaissance pour caractériser l'iconographie de souverains et de généraux modernes : sur un tableau anonyme réalisé vers 1593-1594 faisant partie d'un cycle de treize pièces consacré aux souverains habsbourgeois dans le château Freidegg, en Autriche méridionale, Charles Quint

10. Fernando Checa, Carlos V y la imágen del héroe en el Renacimiento, Madrid, Taurus, 1987, p. 39, ill. 1 - Friedrich Polleross, " "Austriae est imperare orbi universo". Der Globus als Herrschaftssymbol der Habsburger », in W. Krömer (dir.), 1492-1992: Spanien, Österreich und Iberoamerika, actes de colloque, 16 au 21 mars 1992, Innsbruck, Institut für Sprachwissenschaft der Universität Innsbruck, 1993, p. 35 et 41. 
est représenté comme Victor urbis et orbis, couronné de laurier, brandissant un bâton de commandement et se tenant debout sur le globe, entouré de minuscules figures d'ennemis et d'allégories de villes et de pays conquis ${ }^{11}$ (H.-T. 18). Dans la tradition figurative hispano-habsbourgeoise plus tardive, le globe avait surtout pour vocation de renvoyer aux aspirations de domination universelle de la maison d'Autriche, fondées sur l'époque de Charles Quint, où les deux branches des Habsbourg étaient encore unies: un portrait en émail de la famille impériale, par Charles Boit, réalisé au début de la guerre de succession d'Espagne à l'occasion du départ pour Madrid de Charles d'Autriche, le représente en prétendant au trône espagnol, portant devant lui le globe de ses deux mains ${ }^{12}$ (H.-T. 19).

En France même, dans la tradition du portrait de Charles Quint de Parmigianino, l'empereur était représenté en tenant dans la main l'orbe, par exemple sur l'almanach de l'année 1687 qui célébrait la reconquête de la ville de Buda sur les Turcs au début de septembre 1686 (Ill. 2). En revanche, la représentation du monarque français avec le globe était très rare, l'orbe faisant partie des regalia de l'empereur mais aucunement de ceux du monarque français ${ }^{13}$. Sur la statue commandée par Vaini, la représentation de Louis XIV avec le globe à ses pieds devait par conséquent être comprise comme l'appropriation d'un ancien attribut habsbourgeois.

La peau de lion sur laquelle Louis XIV pose son pied droit, dans la statue de Guidi, a également une connotation espagnole. Cet attribut caractérise le personnage représenté, le héros Hercule vainqueur du lion de Némée. C’est une nouvelle fois Charles Quint qui fut le premier

11. Friedrich Polleross, "Sol Austriacus" und "Roi Soleil" - Amerika in den Auseinandersetzungen der europäischen Mächte ", in F. Polleross, A. Sommer-Mathis et Ch. F. Laferl (dir.), Federschmuck und Kaiserkrone: Das barocke Amerikabild in den habsburgischen Ländern, cat. expo., Schloßhof, 10 mai au 13 septembre 1992, Vienne, Bundesministerium für Wissenschaft und Forschung, 1992, p. 54-81.

12. Friedrich B. Polleross, " „Pro decore Majestatis". Zur Repräsentation Kaiser Leopolds I. in Architektur, Bildender und Angewandter Kunst ", Jahrbuch des Kunsthistorischen Museums Wien, $\mathrm{n}^{\circ} 4-5,2002-2003$, p. 190-295.

13. Il n'y a que le globe fleurdelisé qui ait été quelquefois utilisé, voir Matthias Winner, "Le globe comme symbole de l'État dans le cycle de la vie de Marie de Médicis peint par Rubens », in A. Ellenius (dir.), Iconographie, propagande et légitimation, trad. de l'anglais par L. Bury, Paris, Presses universitaires de France, 2001, p. 77-102 - Hendrik Ziegler, "Mazarin et I'image du Roi sous la Fronde", in I. de Conihout et P. Michel (dir.), Mazarin : les lettres et les arts, Paris-Saint-Rémy-en-l'Eau, Bibliothèque Mazarine-Éditions Monelle Hayot, 2006, p. 181-191 - Alexandre Y. Haran, Le lys et le globe :messianisme dynastique et rêve impérial en France à l'aube des temps modernes, Seyssel, Champ Vallon, 2000. 


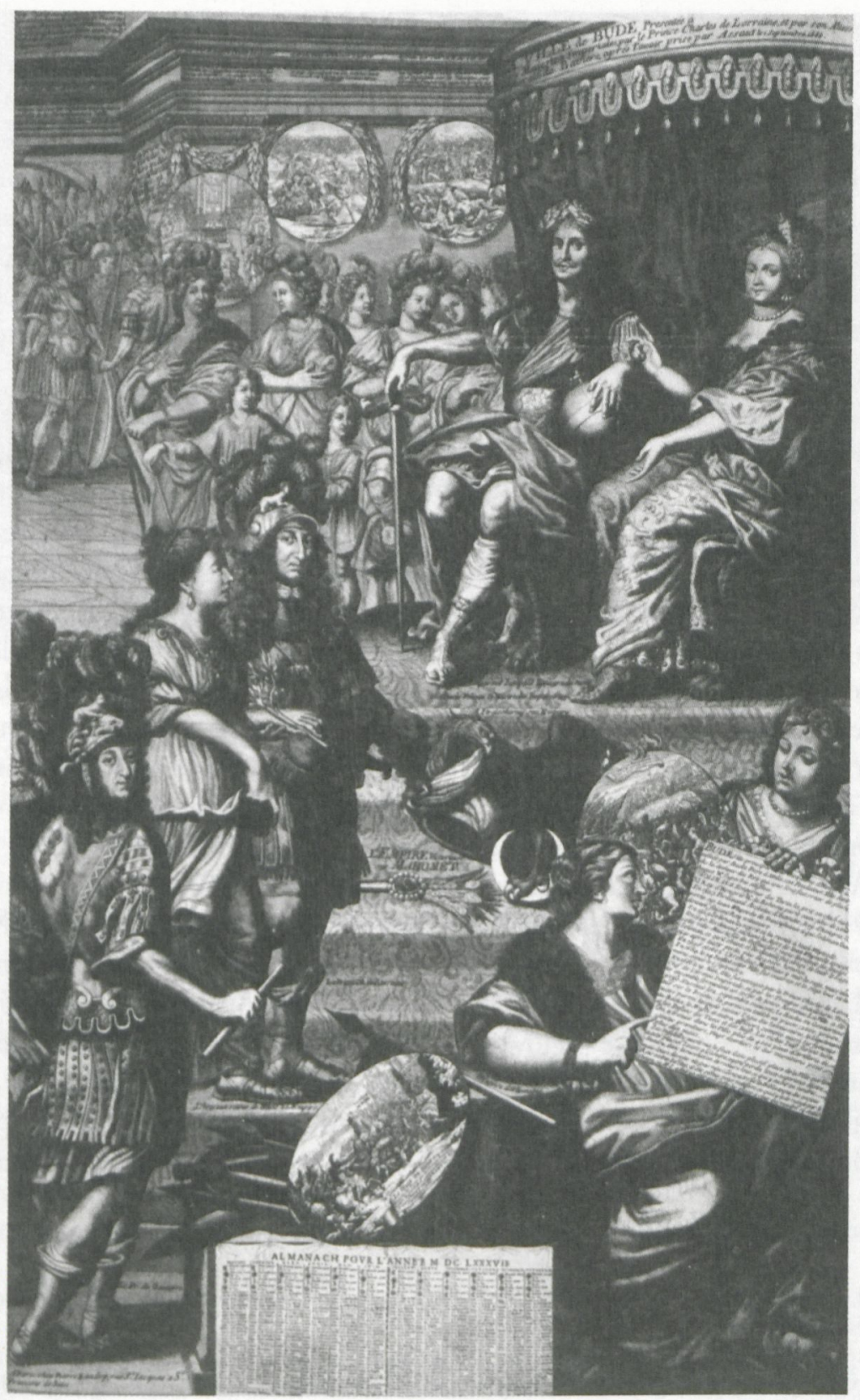

III. 2, Hommage à l'Empereur Léopold ler après la reconquête de Buda, 1686, almanach pour l'année 1687. 
souverain de l'époque moderne à être mis systématiquement sur un pied d'égalité avec le demi-dieu et le héros vertueux des temps mythiques ${ }^{14}$. L'empereur avait choisi les colonnes d'Hercule, situées en Espagne, et la fière inscription "Plvs vltra ", comme devise personnelle ${ }^{15}$. L'identification avec Hercule avait probablement été conçue pour Charles Quint comme un hommage rendu à ses origines espagnoles, puisque, selon Plutarque, Hercule venait d'Espagne. Lidée d'une correspondance privilégiée entre Hercule et le roi d'Espagne était encore vive au XVII ${ }^{\mathrm{e}}$ siècle, bien que l'identification avec le héros antique fût depuis longtemps entrée dans l'iconographie commune des souverains européens ${ }^{16}$.

Dans la statuaire, Louis XIV a été représenté pour la première fois en Hercule vers 1675, par Étienne Le Hongre, sur l'arc de triomphe de la porte Saint-Martin à Paris, érigé pour célébrer les victoires remportées par le roi sur l'Espagne, les Pays-Bas et l'Empire pendant la guerre de Hollande (Ill. 3). Dans une nudité héroïque, mais en perruque d'apparat, avec massue et peau de lion, le monarque triomphe du géant à trois têtes Géryon, à terre, symbole de la Triple Alliance vaincue. Ce parallèle entre les victoires de Louis XIV à la frontière des Pays-Bas espagnols et le dernier des dix travaux d'Hercule avait certainement pour dessein de viser l'Espagne. Le dernier exploit du demi-dieu devait en effet aboutir à sa déification et l'adversaire vaincu, Géryon, roi de Tartessos, avait été, d'après la tradition antique, un souverain de la péninsule ibérique.

Avec la statue romaine de Guidi vingt ans plus tard, le propos n'est plus l'identification à Hercule. Il est frappant de constater que Louis XIV se dresse orgueilleusement sur la peau du lion et sur le globe, la dépouille semblant glisser de celui-ci. Il est vraisemblable que l'on a cherché ici à bafouer une formule figurative héraldique, associée en particulier à Charles II. Déjà sous Philippe IV, la figure du lion posant une patte sur un globe avait été conçue comme un raccourci efficace de l'iconographie du pouvoir de la monarchie espagnole. Dans la salle des Miroirs du palais

14. Guido Bruck, "Habsburger als "Herculier" ", Jahrbuch der kunsthistorischen Sammlungen in Wien, $\mathrm{n}^{\text {elle }}$ série $\mathrm{n}^{\circ}$ 50, 1953, p. 191-198.

15. Kunsthistorisches Museum Wien, Kaiser Karl V. (1500-1558): Macht und Ohnmacht Europas, cat. expo., Kunst- und Ausstellungshalle der Bundesrepublik Deutschland Bonn, 25 février au 21 mai et Kunsthistorisches Museum Wien, 16 juin au 10 septembre 2000, Bonn-Vienne, Vertrieb-Gingko Press-Skira, 2000, p. 162, n 82.

16. G. Bruck, "Habsburger als... ", op. cit., p. 194-196. 


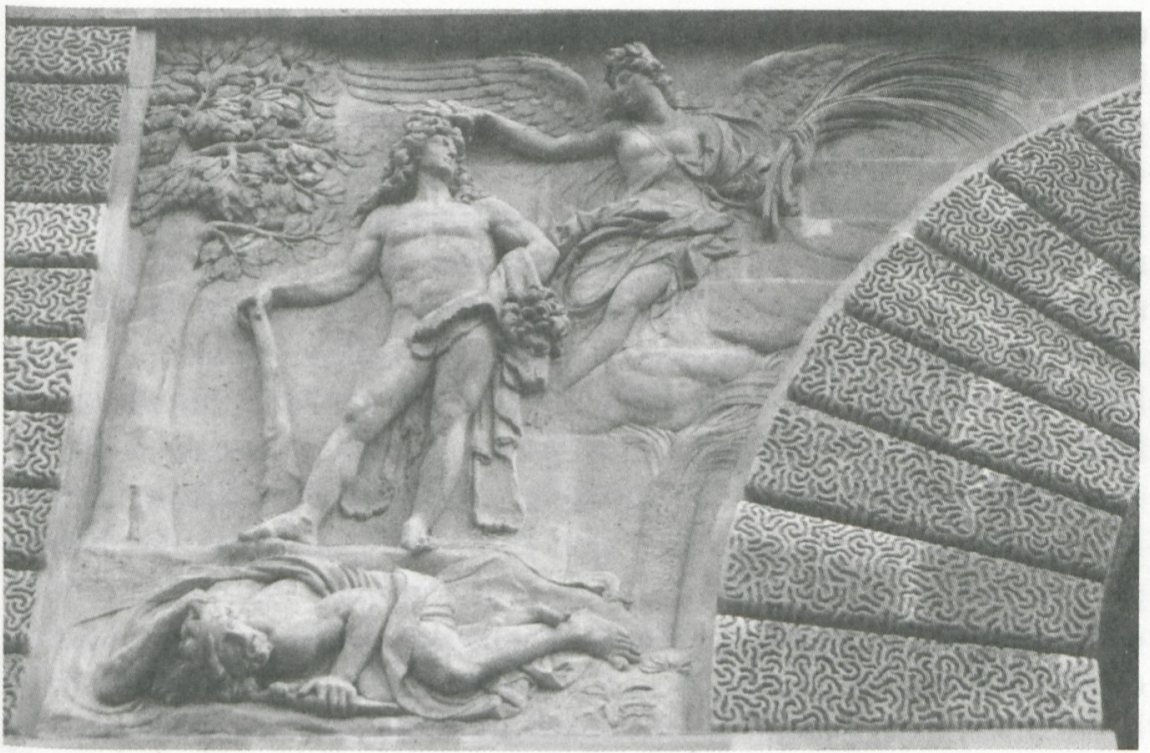

III. 3, Étienne Le Hongre, Louis XIV en Hercule, vers 1674, Paris, porte Saint Martin.

de l'Alcázar, la résidence principale des rois d'Espagne, Philippe IV avait fait installer, sous des miroirs encadrés d'aigles, six imposantes tables de porphyre dont le soubassement était à chaque fois composé de deux lions en bronze doré posant leur patte sur un globe terrestre en marbre. Velázquez avait fait exécuter, sur demande du roi, ces six paires de lions en Italie, pendant son second voyage de 1649 à 1651, pour les expédier en Espagne ${ }^{17}$. Les lions incarnaient la puissance et la vigilance souveraines, les globes sur lesquels ils posaient leurs pattes symbolisaient la revendication de domination universelle exprimée par les Habsbourg d'Espagne ${ }^{18}$. Il n'est donc pas étonnant que le faible Charles II ait été à plusieurs reprises représenté dans une intention politique et propagandiste au début des années 1670 par son peintre de cour, Juan Carreño de Miranda (H.-T. 8), devant l'une de ces tables léonines dans la salle des Miroirs de l'Alcázar

17. Steven N. Orso, Philip IV and the Decoration of the Alcázar of Madrid, Princeton (N. J.), Princeton University Press, 1986, p. 66-67.

18. Miguel Morán Turina, La imagen del rey: Felipe $V$ y el arte, Madrid, Nerea, 1990, p. $21 s q$. 
de Madrid ${ }^{19}$. L'étroite association visuelle de Charles II avec les tables léonines érigées sous le règne de son père entendait souligner sa capacité physique à régner et la légitimité de sa prétention à succéder au trône, qui même en Espagne étaient contestées par son demi-frère, le bâtard Juan d'Autriche, et ses partisans à la cour. En France, ce type de portrait était connu grâce au tableau, également de Carreño, envoyé d'Espagne en 1679 pour les fiançailles de Charles II avec Marie-Louise d'Orléans, qui figurait cette fois le roi en armure de général, tenant dans la main le bâton de commandement, mais toujours devant l'une des tables aux lions de l'Alcázar. Il est significatif de constater que, pendant la guerre de succession d'Espagne, le parti austro-habsbourgeois fit peindre à plusieurs reprises son candidat au trône selon la formule figurative développée par Carreño de Miranda, afin d'appuyer la légitimité des revendications de Charles III. Ainsi en 1703 à Rome, l'émissaire impérial Léopold Joseph, comte de Lamberg, suscita l'irritation du pape en faisant accrocher, pour la célébration de la fête du prétendant autrichien au trône, un portrait de Charles III à côté des armoiries du pape dans l'église de la nation germanique. Après avoir cédé à l'opposition pontificale, le diplomate fit transporter le tableau en un cortège triomphal à sa résidence. Ce portrait était une paraphrase directe des célèbres portraits de Charles II avec les lions et leurs globes dans la salle des Miroirs de l'Alcázar ${ }^{20}$.

La couronne de laurier sur la statue du roi de France commandée par Vaini pouvait elle aussi être ressentie d'un point de vue hispanohabsbourgeois comme une véritable provocation. Certes, la couronne de la victoire faisait partie des signes de distinction plus largement répandus pour un conquérant : Louis XIV en est par exemple couronné sur le relief, déjà évoqué, de la porte Saint-Martin. Associée au globe, la couronne de laurier distinguait cependant, depuis Charles Quint, l'empereur habsbourgeois

19. Museo nacional del Prado, Museo del Prado: inventario general de pinturas. I, la colección real, Madrid, Museo del Prado-Espasa Calpe, 1990, p. 768, n 357.

Nombreuses versions conservées, entre autres à Berlin (1673), à Madrid (1675), au château de Rohrau : Fernando Checa (dir.), Cortes del Barroco: de Bernini y Velázquez a Luca Giordano, cat. expo., Palacio Real de Madrid, 15 octobre 2003 au 11 janvier 2004, Madrid, Patrimonio Nacional Madrid-Sociedad Estatal para la Acción Cutural Exterior, 2003, p. 262, n 71 ; ainsi que dans I'hôtel de ville de Tarragone : Friedrich Polleross, " Hispaniarum et Indiarum Rex. Zur Repräsentation Kaiser Karls VI. als König von Spanien », in J. Jané (dir.), Denkmodelle, actes du colloque hispano-autrichien tenu à Tarragone, 13 au 18 décembre 1999, Tarragone, Universita Rovira i Virgili, 2000, p. 121-173, p. 126, note 18.

20. F. Polleross, " "Pro decore Majestatis"... », op. cit., p. 125-127. 
comme celui qui, parmi les monarques occidentaux situés dans la lignée des empereurs de l'Antiquité, était en droit d'exprimer une revendication de souveraineté universelle ${ }^{21}$.

Il faut retenir qu'avec la statue romaine de Louis XIV, le globe et la peau de lion ne représentent pas seulement les traditionnels attributs de souveraineté impériaux et habsbourgeois, conférés ici au roi français. Dans cette combinaison unique figurée aux pieds du monarque - la flasque peau du lion dépecé glissant du globe - la statue représente davantage une variation, presque ironique, de la formule figurative héraldique, spécialement associée à Charles II d'Espagne. La comparaison de la statue avec le portrait d'État de Carreño de Miranda dans ses différentes versions suggère une telle interprétation.

\section{Les menaces de mort proférées par l'émissaire impérial Martinitz}

Le caractère usurpateur et humiliant que revêtait la statue de Guidi pour la maison de Habsbourg n'avait pas échappé aux contemporains. Dès le mois de septembre 1697, cette étrange statue avait suscité le courroux de l'émissaire impérial, Georg Adam, comte de Martinitz. Il avait joint à un rapport envoyé à Léopold $\mathrm{I}^{\mathrm{er}}$ une Gazetta a mano, document qui réunissait un ensemble d'informations circulant à Rome. L'une de ces nouvelles disait :

Le cardinal Maidalchini étant venu voir la statue du roi de France, que sculptait Domenico Guidi pour le marquis Vaini, dit, après l'avoir considérée, qu'il lui manquait la couronne de laurier sur les tempes. Le sculpteur lui répondit promptement que l'on ne devait en sculpter qu'aux seules statues impériales. Il faut savoir que le marquis Vaini avait commandé à Guidi de sculpter le monde sous le pied gauche et un lion à côté du pied droit. Mais on fit comprendre à l'artiste par un message secret que s'il s'exécutait, il terminerait sa statue dans l'autre monde: le lion figurant sur les armes

21. Ainsi en 1695 sur le buste de marbre de Léopold ler par Paul Strudel, représentant l'empereur avec une perruque d'apparat et une couronne de laurier fortement développée; voir Hubert Glaser et al., Kurfürst Max Emanuel: Bayern und Europa um 1700, cat. expo., Altes und Neues Schloss Schleißheim, 2 juillet au 3 octobre 1976, t. II, Munich, Hirmer Verlag, 1976, p. 34, $n^{\circ} 65$. 
du royaume de Bohême et le monde étant un symbole propre à l'empereur, on en déduit que cette mise en garde provenait de l'émissaire impérial ${ }^{22}$.

Martinitz était l'homme parfait pour une telle opération d'intimidation. Dès le début de son mandat romain, il avait choisi la confrontation avec les partisans de Louis XIV dans la Ville éternelle ${ }^{23}$. Il est significatif que Martinitz n'ait pas signalé à l'empereur la menace de mort reçue par Guidi dans son rapport mais par la revue de presse jointe, ce qui est de toute évidence une mesure de sécurité pour laisser tout lecteur, même un lecteur non autorisé, dans l'incertitude quant à savoir si une telle menace avait été véritablement proférée ou s'il ne s'agissait que d'une rumeur diffamante ${ }^{24}$.

Le cardinal Francesco Maidalchini était l'un des partisans de la France à Rome. Ainsi que d'autres hommes d'église, comme Orsini et Mancini, il obtint de Louis XIV une pension pour avoir défendu les intérêts de la France au sein du collège des cardinaux ${ }^{25}$. Par conséquent, il n'est pas étonnant que Maidalchini ait rendu visite à l'atelier de Guidi après que les premières rumeurs sur des mesures d'intimidation de l'émissaire autrichien eurent commencé à circuler. Il pourrait avoir éventuellement agi sur demande du chargé des affaires françaises, le cardinal de Bouillon, ami de Vaini : il ne devait pas seulement s'informer du niveau de l'avancement des travaux mais également encourager le sculpteur à ne pas se laisser intimider par le parti autrichien. Que des sculpteurs installés à Rome, chargés de la commande de monuments politiquement délicats, aient été contactés par

22. "Il Cardinal Maidalchini essendo stato a vedere La Statua del Rè di Francia, che scolpisce Domenico Guidi per il Marchese Vaini, e doppo haverla considerata, disse, che Li mancava alle Tempie La Corona di Laura, mà Lo scultore accorto gli rispose, che solamente à quelle dé Cesari si doveva scolpire. E qui è dà sapersi, che havendo ordinato il Marchese Vaini al detto Guidi, che ponesse sotto il piede sinistro di questa Statua il Mondo, e vicino al Destro n'intagliasse un Leone, per Biglietto Segreto fù fatto intendere all'Artefice, che ciò non attentasse, perche haverebbe terminata La Sua Statua nell'altro Mondo: ed'essendo il Leone L'Arme del Regno di Boemia, e il Mondo propria Divisa dell'Imperatore, fu creduto, che tal'avviso pervenisse dall'Ambasciator Cesareo. "Österreichisches Staatsarchiv, Vienne, section Haus-, Hof- und Staatsarchiv, Rome, correspondance, carton 78, dossier "Georg Adam Martinitz an Leopold I. (1697 VI 1-XII 21) », fol. 52-61.

23. Concernant d'autres querelles de préséance de Martinitz avec ses collègues français, voir ibid., Rome, correspondance, cartons 79 et 81.

24. Dans sa réponse à Martinitz, Léopold ler n'évoque pas l'incident; voir ibid., Rome, correspondance, carton 81, dossier " Leopold I an Martinitz 1697 I 26 - XII 14 ".

25. Charles Gérin, Louis XIV et le Saint-Siège, t. I : 1655-1667, Paris, V. Lecoffre, 1894, p. $575-576$. 
les différents groupes d'intérêts et en aient subi les pressions, cela, à vrai dire, a été une pratique courante ${ }^{26}$.

Martinitz avait très bien perçu la dimension politique de cette œuvre, qui était à tous égards anti-habsbourgeoise, surtout en ce qui concernait la couronne de laurier et le globe placé sous le pied de Louis XIV, attributs impériaux caractéristiques. Quant au lion, comme Martinitz était issu de la noblesse de Bohême et avait fait des études de droit à Prague, il l'interpréta comme l'emblème de sa patrie, apparemment parce que Léopold I ${ }^{\text {er }}$ était seulement représenté avec cet animal dans sa fonction de roi de Bohême. Pour cette raison, la possible association avec le lion héraldique espagnol lui échappa.

À la fin de l'année, les scandaleuses menaces de mort reçues par Guidi se répandirent comme une traînée de poudre dans les gazettes européennes. Celles-ci signalaient que le sculpteur avait effectivement abandonné son œuvre ${ }^{27}$. Une lettre très détaillée, adressée par le cardinal de Bouillon au roi de France le 19 mai 1699, permet de savoir que la statue, désormais achevée à cette date, avait été terminée non pas par Domenico Guidi mais par un sculpteur français dont le nom était tu. Entre-temps, Vaini avait fait transférer la sculpture de l'atelier de Guidi à son palais, et celle-ci présentait enfin une couronne de laurier sur la perruque d'apparat, ainsi que l'avait exigé le cardinal Maidalchini.

Le zèle de M. le Prince de Vaini pour tout ce qui a raport à la gloire de V. M. luy ayant inspiré le dessein de faire faire icy la Statuë, en marbre, Elle a été achevée ces jours cy. L’ouvrier italien qui la faite avoit été plusieurs fois intimidé par les menaces secretes de l'ambassadeur de l'Empereur pour l'empêcher de mettre le monde sous les pieds de cette statuë, c'est ce qui a obligé M. le Prince de Vaini de la faire achever chez luy par un ouvrier françois; Et le maitre du Sacré Palais n'ayant jamais voulu donner permission depuis le départ de M. de Vaini, de graver cette statuë avec le monde sous les pieds, et une couronne de laurier sur la teste; pretendant mal a propos que l'Empereur est seul en possession d'être representé icy avec cette couronne, J'ay cru devoir engager le graveur qui est françois à passer sur la deffense qui luy étoit faite par le Maitre du Sacré-Palais, sans la permission

26. Sylvia Pressouyre, Nicolas Cordier : recherches sur la sculpture à Rome autour de 1600, t. I, Rome, École Française de Rome, 1984, p. 156.

27. Gazette de Rotterdam, 31 novembre, puis 7 décembre 1697 : Rome, Archivio Segreto Vaticano, Avvisi, vol. 61, fol. 480r et 494r; voir D. L. Bershad, Domenico Guidi..., op. cit., p. 37 , notes 49 et 50 . 
du quel on n'imprime rien à Rome; ce que le graveur a éxécuté comme V. M. le pourons voir dans l'estampe que j'ay l'honneur de luy envoyer, ayant ajouté sur le pied destal, malgré la deffense qu'on luy en faisoit le surnom de Grand qui est dû à V. M. avec tant de justice. Je n'ay pas voulu parler de cette affaire avant que la planche fut tirée, mais je ne pourray m'empêcher de me plaindre au Pape de la couduite du Maitre du Sacré Palais afin qu'a l'avenir Il ne commette plus une pareille faute, qui apparament luy a été inspirée d'ailleurs. La statuë est de sept pies de haut sur un pied destal de quatre pieds. Elle est tres belle et plus ressemblante dans le marbre qu'elle ne l'est dans la gravure. C'est la premiere qui ait été élevée icy à V. M. Et une marque éternelle du dévouement de M. le Prince de Vaini pour V. $\mathrm{M}^{28}$.

Dans sa réponse, Louis XIV confirma la réception de l'estampe et donna raison au cardinal, en rejetant le point de vue de la censure papale prétendant que la couronne de laurier était un attribut privilégié de l'Empereur ${ }^{29}$. Il est clair qu'avec la réalisation de l'estampe le cardinal de Bouillon cherchait à se faire passer auprès de Louis XIV pour un défenseur zélé et loyal des intérêts français, car il ne jouissait plus des pleines faveurs du roi après s'être rangé du côté de l'archevêque de Cambrai, Fénelon, tombé en disgrâce ${ }^{30}$.

Quant au sculpteur qui remplaça Domenico Guidi, intimidé par les menaces de mort proférées par l'émissaire impérial, il s'agit probablement de Pierre Legros le Jeune, natif de Paris, qui séjournait à Rome depuis 1690 comme pensionnaire de l'Académie de France et qui, après avoir obtenu ses premières grandes commandes de la part de l'ordre des Jésuites, s'était définitivement installé comme artiste libre dans la Ville éternelle en $1695^{31}$. En tant qu'ancien pensionnaire de l'Académie, par ailleurs, Legros connaissait assurément Domenico Guidi, qui,

28. Archives du ministère des Affaires étrangères (AAE), Paris, correspondance politique, sous-série : Rome, 401, cardinal de Bouillon à Louis XIV, Rome, 19 mai 1699, p. 120-121.

29. Ibid., Louis XIV au cardinal de Bouillon, Versailles, 10 juin 1699, fol. 126-128; voir D. Erben, Paris und Rome..., op. cit., p. 250, note 61 : "J'ay receu les estampes que vous avez envoyées de la Statue que le Prince de Vaini a fait faire. Si la Couronne de Laurier marquoit quelque jurisdictions dans Rome, les officiers du Pape entendroient mal les interests de Sa Sainteté en voulant reserver cette Eouronne distinction seulement a l'Empereur. " La rayure se trouve dans le document original.

30. Concernant la situation difficile dans laquelle s'est trouvé Bouillon à l'époque, voir son écrit justificateur adressé au roi : AAE, correspondance politique, sous-série : Rome, 401, cardinal de Bouillon à Louis XIV, Rome, 21 avril 1699, fol. 30-34.

31. François Souchal, French Sculptors of the 17th and 18th Centuries: The Reign of Louis XIV, t. II, p. 273 et t. IV, p. 145, Oxford, Cassirer, 1977-1993. 
à la demande de l'Académie, avait de temps en temps dirigé et surveillé les sculpteurs français installés à Rome. Il se pourrait que le cardinal de Bouillon ait attiré l'attention de Vaini sur ce sculpteur déjà d'un certain renom. Immédiatement après son arrivée, le cardinal avait pu, en tant que chargé des affaires françaises à Rome en juin 1697, contacter Legros pour la conception d'un important mausolée comportant un grand nombre de figures, construit en l'honneur de la famille de Bouillon et destiné à l'église abbatiale de Cluny ${ }^{32}$. Ainsi faudrait-il considérer Legros comme le second sculpteur après Guidi ayant participé à la réalisation de la statue du roi commandée en 1697 par Vaini.

\section{L'ancien désir d'une présence sculpturale des rois de France à Rome}

La signification de la réalisation de la sculpture par Vaini - premier monument de Louis XIV à Rome - ne se comprend que dans le contexte de l'ancienne lutte des rois de France pour une présence figurative dans la Ville éternelle, inaugurée pendant le règne d'Henri IV. Dès le début du siècle, l'engagement artistique français à Rome avait été conçu non seulement comme une démonstration de force vis-à-vis du pape, mais surtout comme une réponse aux ambitions de la politique artistique déployée par l'influente faction espagnole dans la ville. Deux cas connus suffiront ici à témoigner de ce climat de surenchère qui contribua à déterminer le succès obtenu par le parti français grâce à la réalisation de la statue du roi par Vaini.

La statue de bronze d'Henri IV, premier roi Bourbon, réalisée entre 1606 et 1609 par Nicolas Cordier et son atelier et commandée par le chapitre de Saint-Jean de Latran, avait accompli pour la première fois l'exploit de célébrer à Rome un souverain régnant, donc encore en vie (Ill. 4). Le pape Paul V Borghèse avait donné son accord au projet, mais, par égard pour la faction espagnole, il avait fait en sorte que l'effet public de ce monument politiquement délicat demeurât limité : il fut ainsi placé dans une niche latérale, fermée par une grille, au rez-de-chaussée de la loggia

32. Ibid., t. II, p. 278-280, $n^{\circ} 9$; Mary Jackson Harvey, "Death and Dynasty in the Bouillon Tomb Commissions », The Art Bulletin, LXXIV, 1992, p. 271-296, en particulier p. 285-287. 


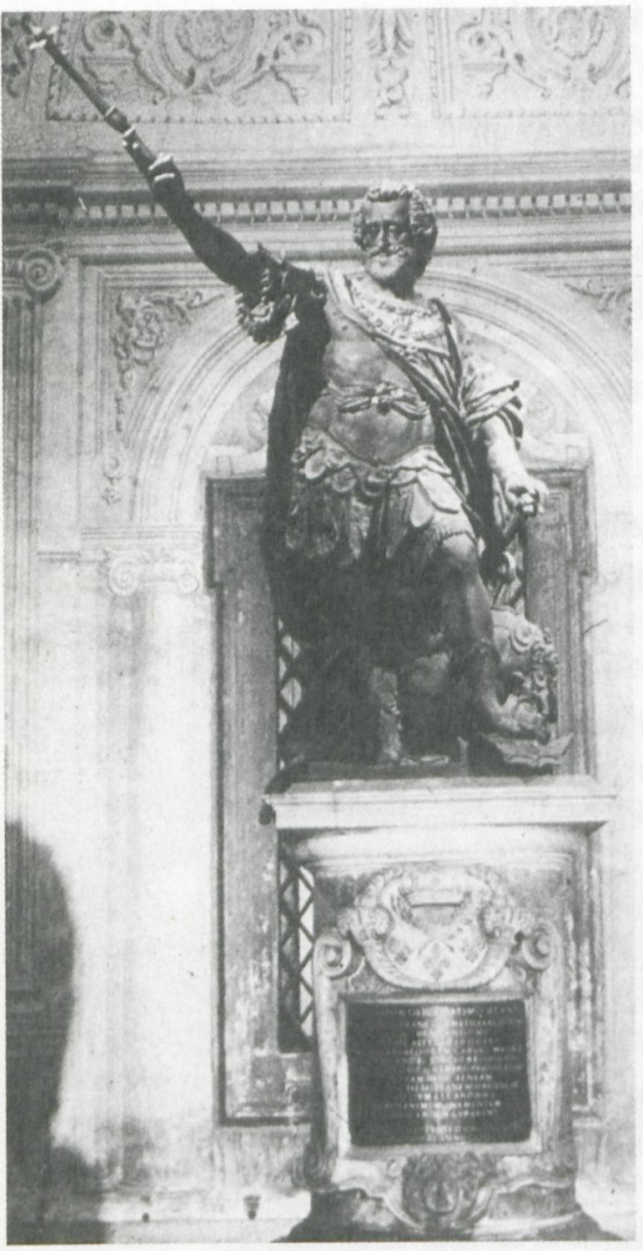

III. 4, Nicolas Cordier et Gregorio De'Rossi, Henri IV, 1606-1609, Rome, Saint-Jean-de-Latran. des Bénédictions de la basilique du Latran; cette situation resta inchangée jusqu'en $1733^{33}$. L'érection d'une statue d'un roi de France provoqua une levée de boucliers de la faction espagnole, qui entendait, dès 1643, faire réaliser aussi une statue de bronze similaire de leur monarque pour l'église de Sainte-MarieMajeure, protégée par la couronne d'Espagne. Pour des raisons financières, le projet prit du retard et ce ne fut que dans les années 16641666 que Girolamo Lucenti, travaillant d'après des croquis de son maitre Gian Lorenzo Bernini, exécuta une statue de bronze de Philippe IV. Dans ce cas également, la visibilité du monument fut très limitée : en 1691 seulement, les chanoines, profitant d'une vacance du siège pontifical, parvinrent à ériger la statue dans le Cortile Grande, situé derrière le palais des chanoines de Santa Maria Maggiore, avant de lui conférer un emplacement plus adéquat en 1692, dans le vestibule de la sacristie de l'église ${ }^{34}$.

Un autre projet monumental français avait ravivé en ces années à Rome la concurrence artistique et politique entre la France et l'Espagne : l'aménagement de l'escalier de la Trinité-des-Monts, par Bernin, présenté pendant l'été 1660 sur demande de Mazarin

33. En 1661 et une nouvelle fois en 1699 , on attira l'attention de Louis XIV sur la mauvaise visibilité de la statue, sans pour autant remédier à cette situation. AAE, correspondance politique, sous-série: Rome, 144, Aubeville à Lionne, Rome, 16 janvier 1662, fol. 59v. Société de l'histoire de l'art français, Correspondance des directeurs de l'Académie de France à Rome avec les surintendants des Bâtiments (1666-1804), édition A. de Montaiglon, t. III, Paris, Charasay Frères, 1887-1908, p. 24-25, n 1017, cardinal de Bouillon à J.-B. Colbert, marquis de Torcy, Rome, 14 décembre 1699.

34. Edward Maser, "The Statue of Henry IV in Saint John Lateran: A Political Work of Art ", Gazette des Beaux-Arts, t. LVI, 1960, p. 147-156 - S. Pressouyre, Nicolas Cordier..., op. cit., t. I, p. 151-158, p. 195-196 et t. II, p. 401-405, n 18 - Steven F. Ostrow, "Gianlorenzo 
et qui prévoyait en son centre l'érection d'une statue équestre de Louis XIV ${ }^{35}$ (Ill. 5). L'ouvrage, prévu non loin de la place d'Espagne et de l'ambassade espagnole, constituait une éclatante provocation vis-à-vis du parti espagnol à Rome, car le sculpteur avait manifestement conçu

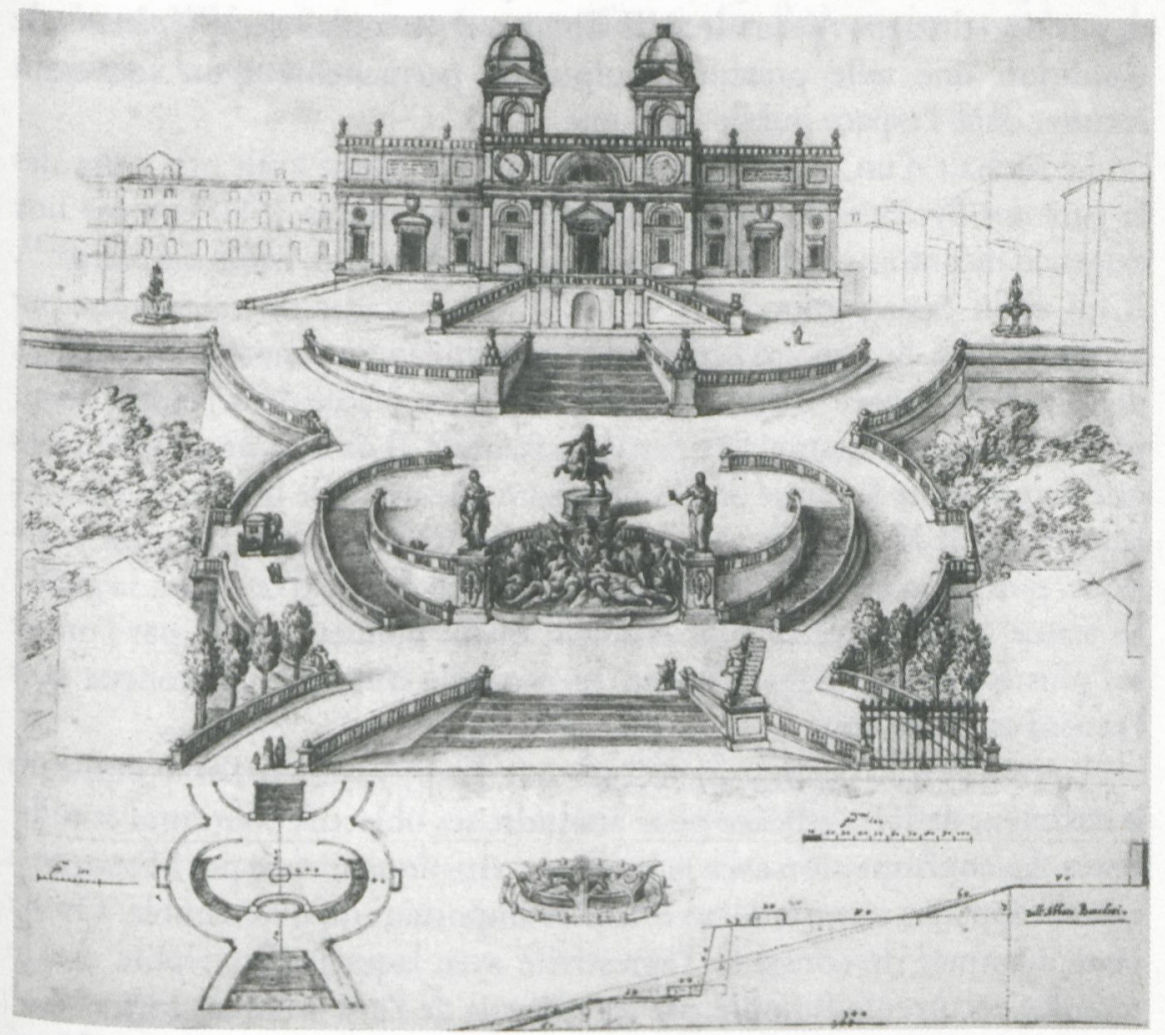

III. 5, Atelier du Bernin (?), projet pour l'escalier de la Trinité-des-Monts, 1660, Bibliothèque apostolique vaticane.

Bernini, Girolamo Lucenti, and the Statue of Philip IV in S. Maria Maggiore: Patronage and Politics in Seicento Rome ", The Art Bulletin, t. LXXIII, 1991, p. 89-118, en particulier p. 112 115 - Diane H. Bodart, "Enjeux de la présence en image : les portraits du roi d'Espagne dans I'Italie du xvı" siècle », in E. Cropper (dir.), The Diplomacy of Art: Artistic Creation and Politics in Seicento Italy, Milan, Nuova Alfa Editoriale, 2000, p. 77-99.

35. Irving Lavin, "Le Bernin et son image du Roi-Soleil ", in J. P. Babelon (dir.), Il se rendit en Italie: études offertes à André Chastel, Rome-Paris, Edizioni dell'Elefante-Flammarion, 1987 , p. 477 , ill. 32 . 
le destrier de son monument équestre en levade en réponse à la statue de Philippe IV, le figurant à cheval dans une posture comparable, réalisée vingt ans plus tôt par Pietro Tacca pour la cour de Madrid. L'œuvre de Tacca, avec laquelle Bernin se serait volontiers mesuré, était l'une des premières fontes en bronze de l'époque à avoir réussi une représentation de ce type ${ }^{36}$. Toutefois, le projet soutenu par Mazarin se heurta à la stricte opposition du pape Alexandre VII Chigi, qui ne voulait accepter à aucune condition une telle présence sculpturale permanente d'un souverain séculier dans l'espace public de Rome.

Le souhait d'un monument de Louis XIV à Rome avait pris corps dès la paix des Pyrénées, et il fut exprimé dans les années 1680 comme une exigence incontournable par François Lemée dans son Traité des Statuës ${ }^{37}$. Il fut enfin exaucé entre 1697 et 1699 avec la statue du roi, voulue par le prince Vaini. Son aboutissement ne fut cependant pas une statue publique, dressée dans l'espace urbain, mais une sculpture placée dans l'espace semipublic d'un palais destiné aux partisans français. Il n'en demeure pas moins que l'exemple de la statue de Guidi illustre une nouvelle fois l'habileté avec laquelle Louis XIV savait gérer les démarches délicates de la politique artistique, sans jamais apparaître comme le commanditaire d'œuvres à sa gloire. Sa statue romaine n'était officiellement qu'un honneur rendu par l'un de ses plus loyaux partisans à Rome, bien qu'elle n'eût pu être conçue sans l'accord tacite du roi.

Louis XIV a su utiliser, mieux que tout autre souverain, l'art comme un instrument de lutte efficace pour atteindre ses objectifs politiques et militaires. La confrontation avec la politique artistique menée par l'Espagne a en cela apparemment joué un rôle plus important qu'il ne semble. On ne peut manquer de constater l'agressivité avec laquelle l'adversaire est de manière récurrente humilié par les moyens de l'art. Mais les fortes réactions de la diplomatie étrangère à la statue du roi commandée par Vaini montrent également que toutes les démarches entreprises par Louis XIV en ce domaine ont été observées avec grande attention. Si, dans leur détail

36. Je remercie le professeur Volker Hunecke, Berlin, de m'avoir signalé un possible rapport de concurrence entre la statue équestre de Tacca et le projet de la Scalinata du Bernin. Voir Volker Hunecke, Europäische Reitermonumente: ein Ritt durch die Geschichte Europas von Dante bis Napoleon, Paderborn, Schöningh, 2008.

37. François Lemée, Traité des Statuës, annoté par D. H. Bodart et H. Ziegler, Weimar, VDG (à paraître) [1688]. 
iconographique, elles ont fait l'objet d'interprétations différentes, leur portée politique a été analysée avec justesse et combattue avec force et pertinence. L'efficacité toute particulière de l'œuvre de Domenico Guidi, sans doute achevée par Pierre Legros le Jeune, qui orne aujourd'hui encore les escaliers de la villa Médicis, consiste dans la capacité d'avoir conféré à la statue l'expression des ambitions de la politique étrangère de Louis XIV, qui ne manqua pas d'atteindre l'adversaire et même de l'inciter à la résistance. 